\title{
Probes for Fundamental QCD Symmetries and a Dark Gauge Boson via Light Meson Decays
}

\author{
Liping Gan*t \\ University of North Carolina Wilmington, Wilmington, NC, USA \\ E-mail: ganl@uncw.edu
}

\begin{abstract}
Light Meson decays provide a unique laboratory to probe fundamental QCD symmetries and to search for new physics beyond the Standard Model. A comprehensive Primakoff experimental program at Jefferson Laboratory (Jlab) is aimed at gathering high precision measurements on the two-photon decay widths and transition form factors at low $Q^{2}$ of $\pi^{0}, \eta$ and $\eta^{\prime}$ via the Primakoff effect. Completed experiments on the $\pi^{0}$ radiative decay width at Jlab $6 \mathrm{GeV}$, and planned measurements of $\eta$ and $\eta^{\prime}$ at Jlab $12 \mathrm{GeV}$ will provide sensitive probes to test the chiral anomaly and to study the origin and dynamics of chiral symmetry breaking. On the other hand, a recently developed Jlab Eta Factory (JEF) experiment will measure various $\eta$ decays with emphasis on rare neutral modes. Compared to all other existing or planned experiments in the world, the JEF experiment offers a clean data set for rare $\eta$ decays to neutral modes with a factor of two orders of magnitude reduction in backgrounds. These results will offer sensitive hadronic probes for weakly-coupled new forces, such as a dark force via a leptophobic dark $V_{B}$-boson or a new $\mathrm{C}$-violating, P-conserving force. An overview of these experimental activities and their physics impacts will be discussed.
\end{abstract}

The 8th International Workshop on Chiral Dynamics, CD2015 ***

29 June 2015 - 03 July 2015

Pisa,Italy

\footnotetext{
* Speaker.

${ }^{\dagger}$ This project is supported by USA NSF awards PHY-1206043 and PHY-1506303.
} 


\section{Introduction}

There are two major challenges in contemporary physics: QCD confinement and new physics beyond the Standard Model (SM). The system of light neutral pseudoscalar mesons $\pi^{0}, \eta$ and $\eta^{\prime}$ provides sensitive probes to investigate both fundamental issues. The symmetries of QCD at low energy are manifested in their most unambiguous form in the sector of light pseudoscalar mesons. While $\pi^{0}$ and $\eta$ are Goldstone bosons due to spontaneous chiral symmetry breaking, $\eta$ is not a Goldstone boson due to the $U(1)_{A}$ anomaly. There is a second type of axial anomaly driving the two-photon decays of $\pi^{0}, \eta$ and $\eta^{\prime}$. This system harbors fundamental information about the effects of isospin or SU(3) symmetry breaking. It opens a new window to determine the fundamental parameters of QCD in a model-independent manner, such as the light quark-mass ratio and the mixing angle of $\eta-\eta^{\prime}$. In addition, $\eta$ is an eigenstate of $\mathrm{P}, \mathrm{C}, \mathrm{CP}$, and $\mathrm{G}\left(I^{G} J^{P C}=0^{+} 0^{-+}\right)$. Its strong and electromagnetic decays are either anomalous or forbidden to the lowest order due to P, C, CP, G-parity and angular momentum conservation. This enhances the relative importance of higher order contributions, making the rare $\eta$ decays a sensitive hadronic probe for weaklycoupled new forces, such as a dark force via a leptophobic dark $V_{B}$-boson or a new C-violating, P-conserving force. Precision measurements of the light pseudoscalar meson decays will provide a unique laboratory to test fundamental symmetries and probe new physics beyond the Standard Model (SM).

The availability of a high duty factor, high precision, continuous electron beam at Jefferson Lab $6 \& 12 \mathrm{GeV}$ offers a great opportunity to perform precision measurements. There have been on-going experimental programs focusing on precision measurements of light mesons at Jlab. An update on the status of these experimental activities is given below.

\section{Primakoff Experimental Program at Jlab}

In the past decade, the PrimEx collaboration has developed a comprehensive experimental program to perform high precision measurements on the two-photon decay widths and transition form factors at low $Q^{2}$ of $\pi^{0}, \eta$ and $\eta^{\prime}$ via the Primakoff effect. The completed experiments on the $\pi^{0}$ radiative decay width (PrimEx-I and PrimEx-II) at Jlab $6 \mathrm{GeV}$, and the other planned measurements at Jlab $12 \mathrm{GeV}$, will offer insight into our understanding of the chiral anomaly, chiral symmetry breaking, and their relationships to the QCD confinement.

\subsection{Neutral pion lifetime measurements at Jlab $6 \mathrm{GeV}$}

The two-photon decay of $\pi^{0}$ reveals one of the most profound symmetry issues in QCD, namely, the explicit breaking of a classical symmetry by the quantum fluctuations of the quark fields when they couple to a gauge field. This phenomenon, called anomalous breaking [1], is of pure quantum mechanical origin and can be calculated exactly to all orders. The predicted anomalous $\pi^{0} \rightarrow \gamma \gamma$ decay width [1][2] is found by expanding the QCD chiral Lagrangian to first order in the pion field, yielding

$$
\Gamma\left(\pi^{0} \rightarrow \gamma \gamma\right)=\left(m_{\pi^{0}} / 4 \pi\right)^{3} /\left(\alpha / f_{\pi}\right)^{2}=7.76 \mathrm{eV},
$$


which has no adjustable parameters. The decay width given above is exact in the chiral limit (except for an experimental uncertainty contribution from the pion decay constant $f \pi=92.21 \pm$ $0.02 \pm 0.14 \mathrm{MeV}$ [3]). Since the mass of the $\pi^{0}$ is the smallest in the hadron spectrum, high order corrections to this prediction due to the non-vanishing quark masses are small $(\sim 4.5 \%)$ and can be calculated with sub-percent accuracy. Stimulated by the PrimEx project, several independent theoretical calculations have been published in the past decade and are shown as the shaded bands in Fig. 1. These calculations are performed either in the framework of chiral perturbation theory $(\mathrm{ChPT})$ up to $\mathscr{O}\left(p^{6}\right)(\mathrm{NLO})[4,5]$ (NNLO corrections are considered in [6]) or based on QCD sum rules [7], and are consistent with an estimated theoretical uncertainty of $\sim 1 \%$. This offers one of the very few unique opportunities to test confinement QCD precisely.

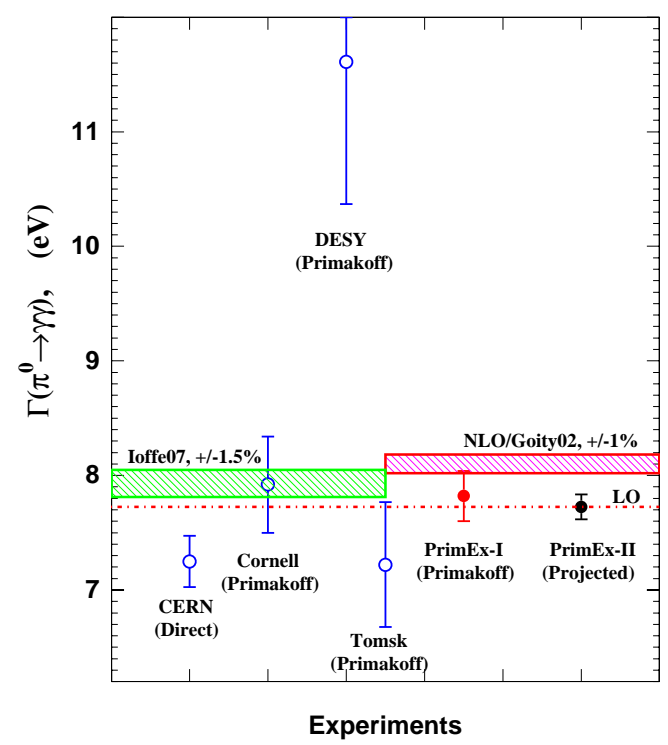

Figure 1: The $\pi^{0} \rightarrow \gamma \gamma$ decay width. The dashed horizontal line is the leading order prediction of the axial anomaly (equation 2.1 [1]). The left hand side shaded band is the recent QCD sum rule prediction [7] and the right hand side shaded band is the next-to-leading order chiral theory predictions $[4,5]$. The experimental results with errors from the left are for: (1) the direct method [9]; (2, $3,4)$ the Primakoff method [10, 11, 12]; (5) published PrimEx-I result [13]. The last point on the right represents the expected error for the PrimExII experiment arbitrarily plotted to agree with the leading order prediction.

The existing experimental results (prior to PrimEx) quoted by the Particle Data Group [8] are shown in Fig. 1 as open circles. These results have large dispersion and their average value is $7.84 \pm 0.56 \mathrm{eV}$ with a $7 \%$ error, which is below the higher order theoretical predictions. In addition, the most precise measurement of the $\pi^{0}$ decay width, prior to the PrimEx experiment, used the direct method of measuring the mean decay length of the $\pi^{0}$ [9]. Their result with the quoted $3.1 \%$ total uncertainty is $\sim 4 \sigma$ lower than the ChPT predictions $[4,5,6]$. The following three data points shown in Fig. 1 were done by using the Primakoff method, however, they have large individual uncertainties. The proposed PrimEx measurement at the $\simeq 1.4 \%$ accuracy level fills this important experimental gap.

The PrimEx experiment measures the $\pi^{0} \rightarrow \gamma \gamma$ decay width via small angle coherent photoproduction of the $\pi^{0}$ in the Coulomb field of a nucleus, i.e. the Primakoff effect [14]. The production of $\pi^{0}$ 's in the Coulomb field of a nucleus by real photons is essentially the inverse of the decay $\pi^{0} \rightarrow \gamma \gamma$, and the cross section for this process thus provides a measure of the $\pi^{0}$ radiative decay width. The Primakoff cross section is peaked at small forward angle and is strongly beam energy dependent $\left(d \sigma_{P r} / d \Omega \sim Z^{2} E^{4} / m^{3}\right)$. Consequently, it requires good angular resolution for the pion production angle in the forward direction, and high precision measurements of the energy 
and the rate of incident photons. Based on these requirements, the PrimEx experiment was optimized to make a significant improvement over the previous Primakoff experiments $[10,11,12]$. This was achieved by a combination of a tagged photon beam and a high resolution, high granularity $\mathrm{PbWO}_{4}$ calorimeter (HYCAL) for detecting the decay photons from $\pi^{0}$. A pair production luminosity monitor was used for photon flux control during the production. The overall systematic uncertainty on the absolute cross section was verified by dedicated measurements of two QED processes: Compton scattering and $e^{+} e^{-}$pair production; it was demonstrated to be better than $1.3 \%$ from the PrimEx-I data analysis.

There were two experiments performed on $\pi^{0}$. The first experiment (PrimEx-I) on two 5\% radiation length (R.L.) targets of ${ }^{12} \mathrm{C}$ and ${ }^{208} \mathrm{~Pb}$ was performed in 2004. The result from PrimEx-I for the $\pi^{0}$ radiative decay width, $\Gamma\left(\pi^{0} \rightarrow \gamma \gamma\right)=7.82 \pm 0.14$ (stat) \pm 0.17 (sys) $\mathrm{eV}$ (shown in Fig. 1), was published in 2011 [13], with a total uncertainty of $2.8 \%$. This result improved the precision of the Particle Data Group average value of $\Gamma\left(\pi^{0} \rightarrow \gamma \gamma\right)$ by more than a factor of two [17]. In order to reach the ultimate goal of $\simeq 1.4 \%$ accuracy on the $\pi^{0}$ lifetime and to test QCD higher order corrections, the second experiment (PrimEx-II) was carried out on two 10\% R.L. targets of ${ }^{12} \mathrm{C}$ and ${ }^{28} \mathrm{Si}$ in fall 2010 , with improved beam quality and charged particle background rejection. The preliminary results of the differential cross sections from the PrimEx-II data are shown in Fig. 2. Two groups are independently analyzing data to extract the $\pi^{0}$ radiative decay width, and the preliminary result of decay width with a total uncertainty of $1.7 \%$ was presented by Gasparian at this workshop. The final result should be expected within a year.
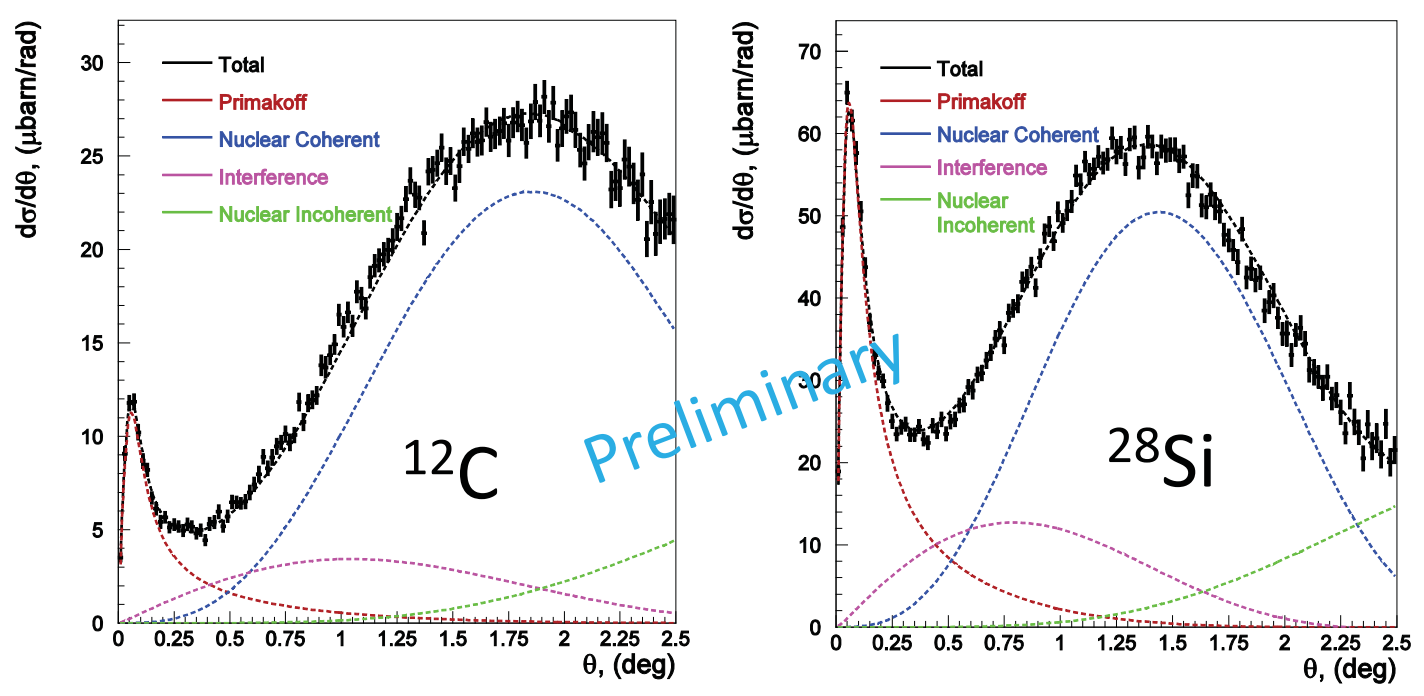

Figure 2: Preliminary PrimEx-II results on the $\pi^{0}$ differential cross sections on the ${ }^{12} \mathrm{C}$ (left) and ${ }^{28} \mathrm{Si}$ (right) targets. The red dashed curves are extracted Primakoff cross sections.

\subsection{Primakoff experiments at Jlab $12 \mathrm{GeV}$}

The $12 \mathrm{GeV}$ Primakoff experimental program $[15,16]$ includes measurements of the two- 
photon decay widths of $\eta$ and $\eta^{\prime}$ and the transition form factors of $\pi^{0}, \eta$ and $\eta^{\prime}$ at the square of four-momentum transfer $Q^{2}$ of $0.001-0.5 \mathrm{GeV}^{2} / \mathrm{c}^{2}$. As described in the previous section, the $\pi^{0}$ decay offers the most sensitive probe to the chiral anomaly and spontaneous chiral symmetry breaking in QCD. On the other hand, due to larger masses, $\eta$ and $\eta^{\prime}$ decays are more sensitive to the breakings of $\mathrm{SU}(3)$ and isospin by the unequal quark masses.

$\mathrm{SU}(3)$ breaking is primarily manifested by $\eta$ mixing with $\eta^{\prime}$, which contributes significantly in the next-to-leading order term in ChPT calculations. Precision measurements of $\eta$ and $\eta$ radiative decay widths will be critical input to determine the $\eta-\eta^{\prime}$ mixing angle. All partial decay widths in the $\eta$ sector are experimentally determined by using the $\eta$ radiative decay width and their corresponding Branching Ratios (BR). Therefore an accurate result for $\Gamma(\eta \rightarrow \gamma \gamma)$ will have a significant impact on the determination of the $m_{d}-m_{u}$ quark mass difference by improving the precision of the $\eta \rightarrow 3 \pi$ decay width $[25,26]$. The measurements of $\eta^{\prime}$ will lead to a better understanding of the $\eta^{\prime}$ mass puzzle, and its nature in general.

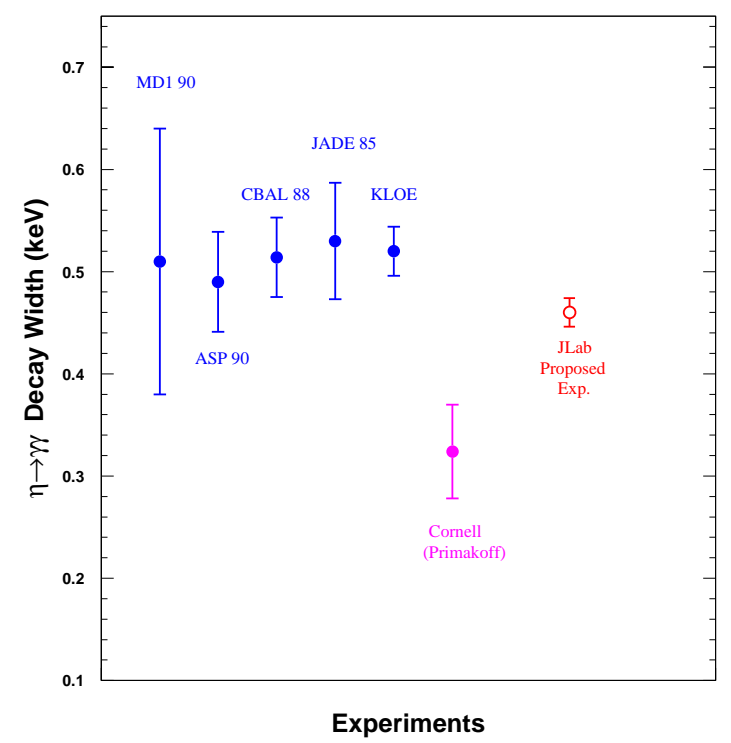

Figure 3: Experimental results of $\Gamma(\eta \rightarrow \gamma \gamma)$. Five points from the left are the results from collider experiments [18, 19, 20,21, 22], point 6 is the result of Cornell Primakoff experiment [27]. Point 7 is the expected error for the proposed PrimEx measurement with $3 \%$ total error, arbitrarily plotted to agree with the average value of previous measurements.

An approved experiment to measure $\Gamma(\eta \rightarrow \gamma \gamma)($ E12-10-011) [23, 24] is currently under preparation at Jlab. The goal of this experiment is to measure the $\eta$ radiative decay width to a $\sim 3 \%$ precision via the Primakoff effect on two light targets, hydrogen and ${ }^{4} \mathrm{He}$, using the newly developed GlueX apparatus in Hall D. ${ }^{1}$ The previous measurements shown in Fig. 3 were performed using two photon interactions either through $e^{+} e^{-}$collisions $\left(e^{+} e^{-} \rightarrow \gamma^{*} \gamma^{*} e^{+} e^{-} \rightarrow \eta e^{+} e^{-}\right)$or via the Primakoff effect. There is a significant difference (at the $3 \sigma$ level) between these two types of measurements. The proposed PrimEx measurement is aimed to resolve this long standing discrepancy by a new Primakoff experiment. Figure 4 and Figure 5 demonstrate how much the projected new measurement of $\Gamma(\eta \rightarrow \gamma \gamma)$ at $3 \%$ precision could improve the $\eta-\eta^{\prime}$ mixing angle and the light quark mass ratio $\mathscr{Q}^{2}=\frac{m_{s}^{2}-\hat{m}^{2}}{m_{d}^{2}-m_{u}^{2}}$, where $\hat{m}=\left(m_{u}+m_{d}\right) / 2$.

The measurements of transition form factors of $\pi^{0}, \eta$ and $\eta^{\prime}$ at small $Q^{2}$, as shown in Fig. 6 and Fig. 7 as examples, will model independently determine the electromagnetic interaction radii

\footnotetext{
${ }^{1}$ A $2 \%$ accuracy could be achieved with future Forward Calorimeter upgrade.
} 


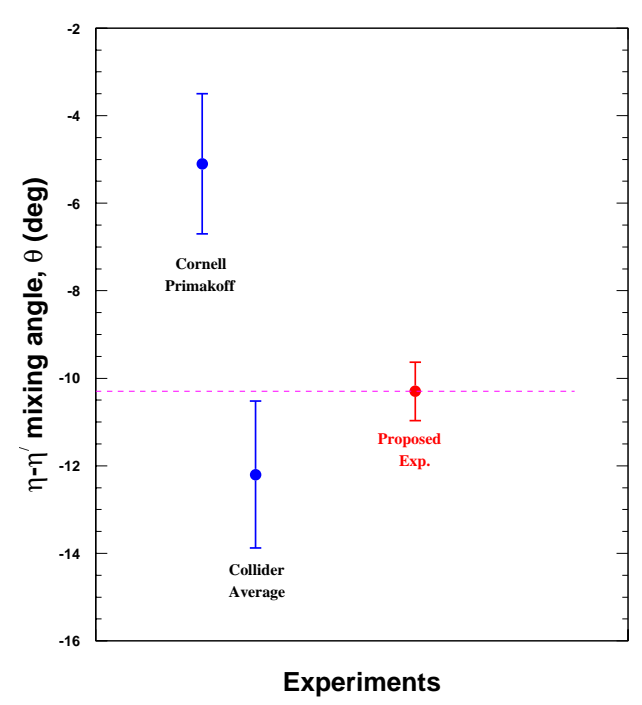

Figure 4: The mixing angles extracted from different experiments. The left two points are mixing angles calculated using the Cornell Primakoff [27] and average from the $e^{+} e^{-}$collider experiments [3]. The expected result from the proposed experiment is arbitrarily set equal to the average value from the two types of existing experiments.

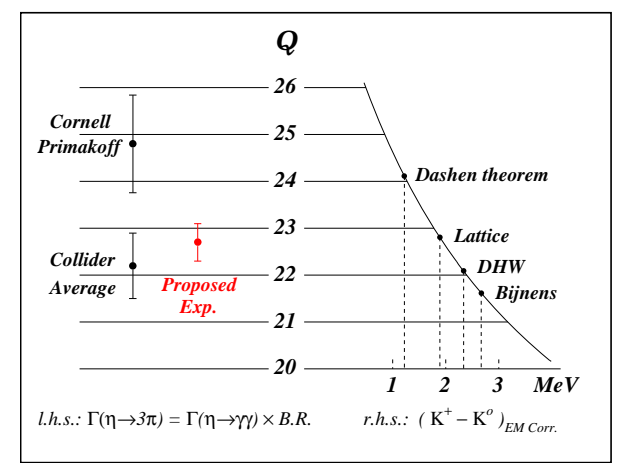

Figure 5: Light quark mass ratio determined by two different methods. The l.h.s. indicates the values of $\mathscr{Q}$ calculated from the $\eta \rightarrow 3 \pi$ decay corresponding to the Primakoff and collider experimental results for the $\Gamma_{\eta \rightarrow \gamma \gamma}$ as input. The r.h.s. shows the results for $\mathscr{Q}$ obtained from the kaon mass difference with four different theoretical estimations for the electromagnetic correction. Taken from Ref. [25].

of those mesons. As pointed by authors of [28, 29], the meson transition form factors at small and intermediate $Q^{2}$ dominate the contributions to the hadronic light-by-light corrections to the anomalous magnetic moment of the muon. The $Q^{2}$ ranges of $0.001-0.5 \mathrm{GeV}^{2} / \mathrm{c}^{2}$ projected by the Jlab Primakoff experiments are complimentary to all existing and planned measurements at relatively larger $Q^{2}$.

\section{Determination of the Quark Mass Ratio via $\eta \rightarrow 3 \pi$}

The fundamental parameter $\mathscr{Q} \equiv\left(m_{s}^{2}-\hat{m}^{2}\right) /\left(m_{d}^{2}-m_{u}^{2}\right)$, with $\hat{m} \equiv\left(m_{u}+m_{d}\right) / 2$, drives isospin violation in SM. Since symmetry forbids a contribution directly proportional to QCD $_{\text {or even to }}$ $m_{u}+m_{d}$, there are only a few strong interaction observables that are sensitive to the quark mass difference. In most cases these isospin-violating observables are also affected by electromagnetic effects, so that if one wants to extract information on $m_{d}-m_{u}$, one has to first calculate and disentangle the contribution due to electromagnetic interactions. For example, in the case of the $K^{+}-K^{0}$ mass difference as shown in r.h.s. of Figure 5, this is problematic and it is therefore difficult to extract phenomenological information on $m_{d}-m_{u}$.

By contrast, the $\eta \rightarrow 3 \pi$ decay is due to almost exclusively to the isospin symmetry breaking part of the Hamiltonian $\sim\left(m_{d}-m_{u}\right)(u \bar{u}-d \bar{d}) / 2$. Moreover, Sutherland's theorem [31, 32] forbids 


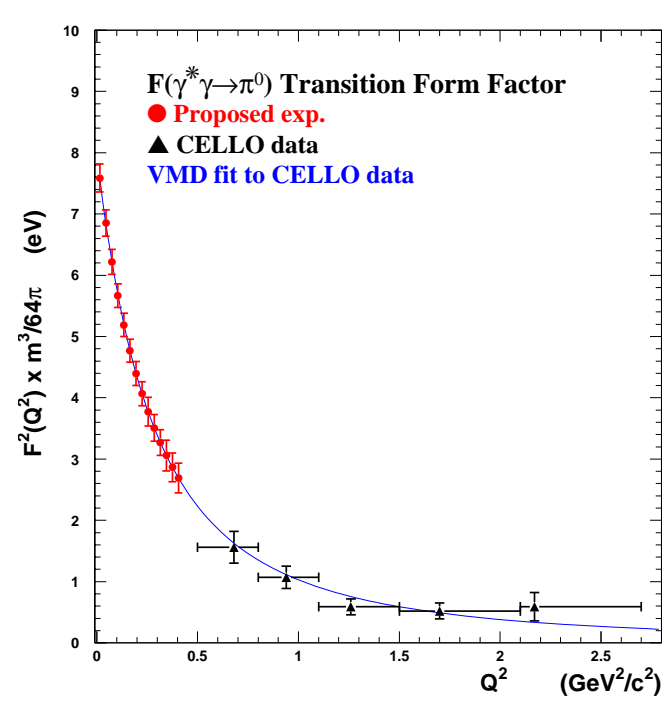

Figure 6: The $\pi^{0}$ transition form factor. The proposed points are projected to the VMD prediction with expected total errors. CELLO data are from reference [30].

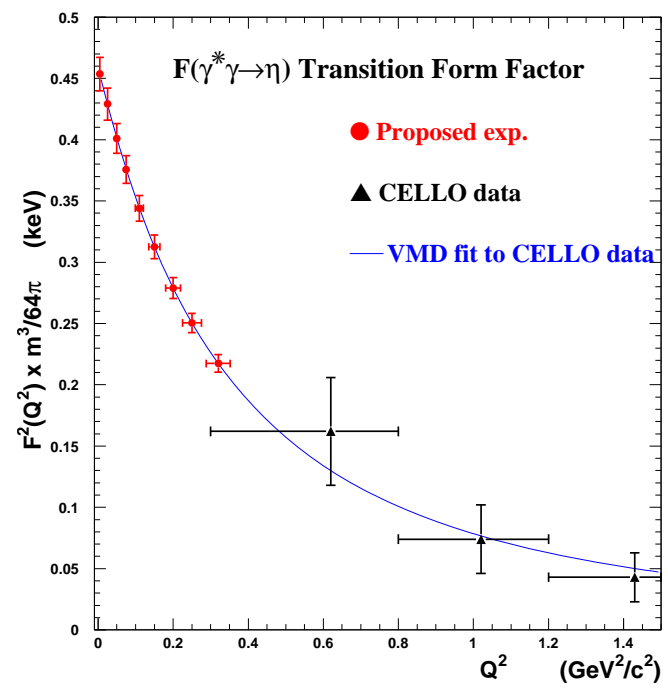

Figure 7: The $\eta$ transition form factor. The proposed points are projected to the VMD prediction with expected total errors, in comparison with CELLO data [30].

electromagnetic contributions in the chiral limit; contributions of order $\alpha$ are also suppressed by $\left(m_{u}+m_{d}\right) / \Lambda_{\mathrm{QCD}}$. This singles out this decay as the best potential source of phenomenological information on the up-down quark mass difference [25, 26]. The amplitude of $\eta \rightarrow 3 \pi$ is proportional to $m_{d}-m_{u}$ and is given by

$$
A(s, t, u)=\frac{1}{\mathscr{Q}^{2}} \frac{m_{K}^{2}}{m_{\pi}^{2}} \frac{\mathscr{M}(s, t, u)}{3 \sqrt{3} F_{\pi}^{2}}, \quad \mathscr{Q}=\frac{m_{s}^{2}-\hat{m}^{2}}{m_{d}^{2}-m_{u}^{2}}, \hat{m}=\left(m_{u}+m_{d}\right) / 2
$$

where $\mathscr{M}(s, t, u)$ is a dimensionless factor that is theoretically calculable, and $s, t$ and $u$ are Mandelstam variables.

To determine the quark mass ratio $\mathscr{Q}$, one needs both experimental measurement of the decay width $\Gamma(\eta \rightarrow 3 \pi)$ and theoretical calculation of $\mathscr{M}(s, t, u)$. The $\Gamma(\eta \rightarrow 3 \pi)$ is determined using the measured BR and the decay width of the normalization channel $\Gamma(\eta \rightarrow \gamma \gamma)$. The approved Primakoff experiment (E12-10-11) described in the previous section 2.2 will measure $\Gamma(\eta \rightarrow \gamma \gamma)$ at $\sim 3 \%$ precision, which will make a significant improvement in $\mathscr{Q}$ as shown in the l.h.s. of Figure 5. On the theoretical side, a precise calculation of $\mathscr{M}(s, t, u)$ is challenging because the chiral expansion for this decay amplitude does not converge very fast. The main difficulty is the rescattering effects of pions in the $S, I=0$ wave. The best solution at hand is to combine ChPT with a dispersion analysis. Some unknown constants, called subtraction constants, enter the calculation and can be fixed from a fit to the experimental Dalitz distribution for $\eta \rightarrow 3 \pi$. The uncertainty due to the experimental Dalitz distributions makes the largest contribution to the total error budget of $\mathscr{Q}$ after $\Gamma(\eta \rightarrow \gamma \gamma)[33,34]$. The measurement of the $\eta \rightarrow 3 \pi$ Dalitz distributions in the JEF experiment, which will be discussed in the next section 4 , will increase the world dataset for the $\eta \rightarrow 3 \pi$ Dalitz distributions in both the charged and neutral channels by a factor of $\sim 3$ with 
improved systematic uncertainties. Combined measurements of both $\Gamma(\eta \rightarrow \gamma \gamma)$ and the Dalitz distributions of $\eta \rightarrow 3 \pi$ will offer an clean determination of the quark mass ratio $\mathscr{Q}$.

\section{The Jlab Eta Factory (JEF) Experiment}

The JEF experimental program "Eta Decays with Emphasis on Rare Neutral Modes: The Jlab Eta Factory (JEF) Experiment" (PR12-14-004) [35, 36] has been recently developed in Hall D. Table 1 summarizes various $\eta$ decays in the scope of JEF program and the physics highlight. The proposed measurements will increase the world dataset for the $\eta \rightarrow 3 \pi$ Dalitz distributions in both the charged and neutral channels by a factor of $\sim 3$ with improved systematic uncertainties by using the standard GlueX apparatus. With an upgraded Forward Calorimeter (FCAL-II), the upper limits of branching ratio for rare $\eta$ decays to neutral modes will be improved by up to two orders of magnitude, and for the first time a sufficient sensitivity will be achieved to map the Dalitz distribution of $\eta \rightarrow \pi^{0} \gamma \gamma$ to probe the scalar meson dynamics in ChPT. Compared to all other facilities in the world, the JEF experiment offers the cleanest dataset for rare $\eta$ decays to neutral modes with a factor of $\sim 2$ orders of magnitude reduction in backgrounds, which is historically the main challenge in rare decays. This will be achieved by the facts that significantly boosted $\eta$ 's are produced through $\gamma+p \rightarrow \eta+p$ with a $12 \mathrm{GeV}$ tagged photon beam, the recoil protons are detected with GlueX detector to reject the non-coplanar backgrounds, and the decay photons are measured in an upgraded forward calorimeter (FCAL-II) with a central region of high-granularity, highresolution lead tungstate crystal insertion. The priority physics campaigns for the JEF experiment are described below.

\subsection{Search for a sub-GeV leptophobic gauge boson $V_{B}$}

Dark Matter (DM) dominates the matter density in the Universe. Understanding its nature provides one of the strongest motivations for physics beyond SM. The stability of DM suggests that there may be a dark sector consisting of rich symmetry structure with new forces and new particles. Discovery of any of these particles, new forces, and associated symmetries would have significant importance in physics. Additional $U(1)^{\gamma}$ gauge symmetry is one of the best motivated extensions of the Standard Model (SM) [38]. A conserved charge can explain the stability of dark matter [39]-[43]. Since the corresponding current conserves all approximate SM symmetries, the coupling strength of DM to SM may exceed the SM weak scale without immediately running into strong constrains imposed by flavor physics and tests of discrete symmetries [44].

One model in the "Vector" portal from the SM sector into the dark sector that has been widely considered is a new force mediated by an abelian $U(1)^{Y}$ gauge boson $A^{\prime}$ (dark photon) that couples very weakly to electrically charged particles through "kinetic mixing" with the photon [45]. Searching for a sub-GeV $A^{\prime}$ has drawn world-wide attentions in recent years and has inspired a broad experimental programs in the high-intensity frontier centers [46]. Most of experimental searches for the $A^{\prime}$ are through its decays to $e^{+} e^{-}$or $\mu^{+} \mu^{-}$, which rely on the leptonic coupling of this new force.

Another equally compelling model in the "Vector" portal not covered by the dark photon searches is a new force mediated by a leptophobic gauge $V_{B}$-boson that couples predominantly to quarks and arises from a new $U(1)_{B}$ baryon number gauge symmetry $[47,48]$. The $U(1)_{B}$ 


\begin{tabular}{|c|c|c|c|}
\hline Mode & Branching Ratio & Physics Highlight & Photons \\
\hline \hline priority: & & & \\
\hline$\pi^{0} \gamma \gamma$ & $(2.7 \pm 0.5) \times 10^{-4}$ & $\chi$ PTh at $\mathscr{O}\left(p^{6}\right)$ & 4 \\
\hline$\gamma+V_{B}$ & beyond SM & leptophobic dark boson & 4 \\
\hline $3 \pi^{0}$ & $(32.6 \pm 0.2) \%$ & $m_{u}-m_{d}$ & 6 \\
\hline$\pi^{+} \pi^{-} \pi^{0}$ & $(22.7 \pm 0.3) \%$ & $m_{u}-m_{d}, \mathrm{CV}$ & 2 \\
\hline $3 \gamma$ & $<1.6 \times 10^{-5}$ & $\mathrm{CV}, \mathrm{CPV}$ & 3 \\
\hline ancillary: & & & \\
\hline $4 \gamma$ & $<2.8 \times 10^{-4}$ & $<10^{-11}[37]$ & 4 \\
\hline $2 \pi^{0}$ & $<3.5 \times 10^{-4}$ & $\mathrm{CPV}, \mathrm{PV}$ & 4 \\
\hline $2 \pi^{0} \gamma$ & $<5 \times 10^{-4}$ & $\mathrm{CV}, \mathrm{CPV}$ & 5 \\
\hline $3 \pi^{0} \gamma$ & $<6 \times 10^{-5}$ & $\mathrm{CV}, \mathrm{CPV}$ & 6 \\
\hline $4 \pi^{0}$ & $<6.9 \times 10^{-7}$ & $\mathrm{CPV}, \mathrm{PV}$ & 8 \\
\hline$\pi^{0} \gamma$ & $<9 \times 10^{-5}$ & $\mathrm{CV}$, & 3 \\
& & Ang. Mom. viol. & \\
\hline normalization: & & & \\
\hline $2 \gamma$ & $(39.3 \pm 0.2) \%$ & anomaly, $\eta-\eta^{\prime}$ mixing & \\
\hline
\end{tabular}

Table 1: The $\eta$ decays highlighted in the JEF program, plus related ancillary channels. The normalization channel $\eta \rightarrow \gamma \gamma$ will be measured by the eta Primakoff experiment (E12-10-011) described in Section 2.2.

local gauge symmetry was initially proposed by Lee and Yang back in 1955 [49] and subsequently discussed extensively in the literature $[43,47,48,50,51]$. A new $U(1)_{B}$ gauge symmetry provides a natural frame-work for the Peccei-Quinn mechanism in the quark sector for solving a long standing "strong CP problem" [50]. This model has also been motivated in part by the similar cosmological abundances of dark matter and baryonic matter in the Universe, which may point toward a unified baryogenesis mechanism for both types of matter [54]. New baryonic fermions with electroweak quantum numbers are required to cancel the $S U(2)_{L}^{2} \times U(1)_{B}$ and $U(1)_{Y}^{2} \times U(1)_{B}$ anomalies. The new fermions acquire masses $(\Lambda)$ via a $U(1)_{B}$-breaking Higgs field, with $m_{B} / \Lambda \geq g_{B} /(4 \pi)$ [55], where $m_{B}$ is the mass of $V_{B}$-boson and $g_{B}$ is the $U(1)_{B}$ gauge coupling.

The experimental signatures for $V_{B}$ depend on its mass $m_{B}$ and decay channels. Recently there are several new initiatives in the field. For the scenarios in which $V_{B}$ decays to dark matter (invisible decay), Pospelov et al. suggested to search for a sub-GeV $V_{B}$ at neutrino factories such as MiniBooNE with a projected sensitivity to baryonic fine structure constant down to $o_{B} \sim 10^{-6}$ [44]. On the other hand, a very recent proposal is aimed to explore a possible $V_{B}$ at a few $\mathrm{TeV}$ mass range in the semivisible jets at LHC [53]. In the case of the dark matter particle mass $m_{\mathcal{C}}>m_{B} / 2$, it is more sensitive to search for $V_{B}$ directly through observation of the visible final states [44], which is a main focus of the JEF experiment described below.

Experimental searches for leptophobic bosons at hadron colliders over the last few decades have set upper limits on their couplings for masses in the $50 \mathrm{GeV}$ to $3 \mathrm{TeV}$ range $[52,56,57]$. 
Masses smaller than the pion mass also have very strong constraints from searches for long-range nuclear forces [58]. However, masses around the QCD scale have been nearly "untouched" due to large SM backgrounds [52]. Nelson and Tetradis first proposed to search for a sub-GeV $V_{B}$-boson through the $\eta$ decay in 1989 [47]. They assumed that $V_{B} \rightarrow \pi^{+} \pi^{-}$would dominate for $m_{B}>2 m_{\pi}$. In that case, the signal of $V_{B}$ would be mostly hidden under the $\rho$ meson decay. Tulin demonstrated in his recent article [48] that $V_{B} \rightarrow \pi^{+} \pi^{-}$is suppressed due to $\mathrm{G}$ parity conservation and the leading decay channel is $V_{B} \rightarrow \pi^{0} \gamma$ for $m_{\pi} \leq m_{B} \leq 620 \mathrm{MeV}$. This offers a unique experimental opportunity to search for $V_{B}$ in this mass range through the doubly-radiative decay $\eta \rightarrow \pi^{0} \gamma \gamma$. The new physics decay $\eta \rightarrow V_{B} \gamma \rightarrow \pi^{0} \gamma \gamma$ would produce a resonance peak at $m_{B}$ in the $\pi^{0} \gamma$ invariant mass distribution, while the SM-allowed $\eta \rightarrow \pi^{0} \gamma \gamma$ decay with a suppressed branching ratio of $\sim 2.7 \times 10^{-4}[56]$ would be present as the irreducible background in the signal window.

The JEF experiment will search for $V_{B}$-boson in the mass range of $0.14-0.54 \mathrm{GeV}$ through $\eta \rightarrow \gamma+V_{B}\left(\rightarrow \gamma \pi^{0}\right)$. The experimental sensitivity with 100 days of beam time is shown in Fig. 8 . This measurement will improve the existing bounds by two orders of magnitude, with sensitivity to the baryonic fine structure constant $\alpha_{B}$ as low as $10^{-7}$, indirectly constraining the existence of anomaly cancelling fermions at the TeV-scale. The JEF search for a $V_{B}$-boson is complementary to other accelerator-based searches for invisible decays, such as newly proposed program at the MiniBooNE neutrino factory [44]; it is also complementary to ongoing worldwide effort for a dark photon focusing mainly on signatures involving leptons.

\subsection{Search for a C-Violating, P-Conserving (CVPC) interaction}

The CP violation in the SM fails about ten orders of magnitude to account for the observed dominance of matter over anti-matter. Searching for new sources of CP violation is therefore a high priority. Although $\mathrm{C}$ is generally assumed to be an exact symmetry in the non-weak interactions, however, it has not been well tested and the current experimental limit is only at $0.3 \%-0.5 \%$ level for the amplitude [61]. The SM forbidden $\eta$ decays into pions and photons represent some of the few opportunities nature has provided to directly test $\mathrm{C}$ invariance in the P-conserving reactions.

As pointed out by Ramsey-Musolf [33, 62, 63], CVPC interactions are a largely unexplored area of fundamental symmetry tests. Analyzing their effects for light quark systems requires an Effective Field Theory (EFT) approach, as they do not arise at tree-level via renormalizable gauge interactions. Electric Dipole Moment (EDM) measurements test $\mathrm{CP}$ violation accompanied by P violation, indirectly constraining CVPC via Electro-Weak (EW) radiative corrections from the standpoint of short distance parity restoration and/or naturalness. In general, EDM measurements place stringent constraints on new CVPC interactions. Exceptions may exist in the presence of a conspiracy or new symmetry at the T-violating and P-conserving (TVPC) matching scale [33, 62] (equivalent to CVPC due to the CPT theorem). If parity remains broken at short distances, the experimental EDM limits do not constrain the existence of a new CVPC interaction. Since the mass hierarchy is a priori unknown (ie, whether the scale of $\mathrm{C}$ invariance restoration is above or below the scale of P invariance restoration), only direct searches for the CVPC interactions such as proposed measurements in the $\eta$ decays are unambiguous [63].

The JEF experiment will measure the C-violating $\eta$ decays such as $\eta \rightarrow 3 \gamma$ and $\eta \rightarrow 2 \pi^{0} \gamma$ to reduce the upper limits of branching ratio by up to 1.5 orders of magnitude. In the meantime, a clean dataset of $\eta \rightarrow \pi^{0} \pi^{+} \pi^{-}$will offer an opportunity to test $\mathrm{C}$ violation by searching for the 


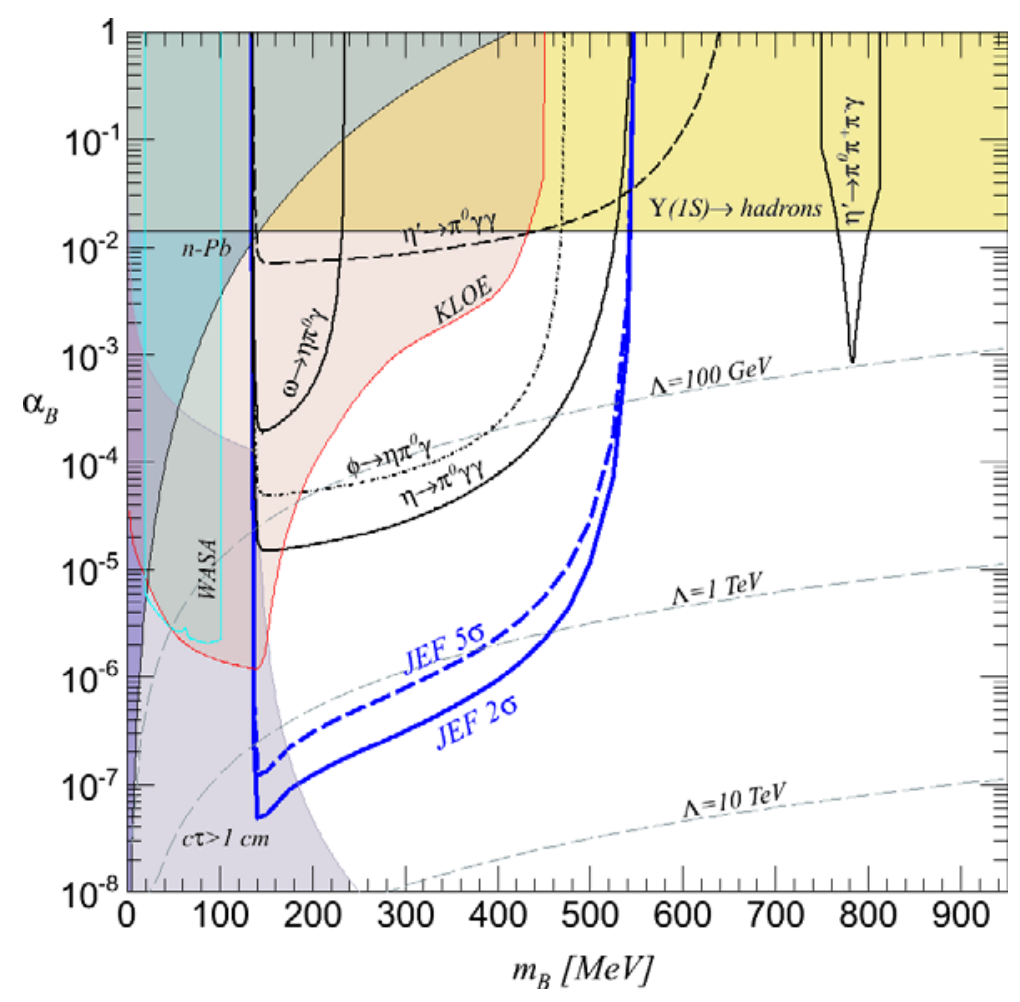

Figure 8: Current exclusion regions for a leptophobic gauge $V_{B}$-boson [48], with the projected JEF search region for the baryonic fine structure constant versus mass plane. Shaded regions are exclusion limits from hadronic $\Upsilon(1 S)$ decay [57] and low energy $n-P b$ scattering [58]. The pink and blue shaded regions are from the dark photon $A^{\prime}$ searches (KLOE [59] and WASA [60]). The $A^{\prime}$ limits applied to $V_{B}$ are modeldependent, constraining possible $V_{B}$ leptonic couplings. Limits shown here are for the leptonic couplings of $\varepsilon=0.1 \times e g_{B} /(4 \pi)^{2}$. The black contours are current exclusion limits from radiative light meson decays based on their total rate (assuming the QCD contribution is zero). The light purple shaded region shows where $V_{B}$ has a macroscopic decay length $c \tau>1 \mathrm{~cm}$. The solid blue curve shows the projected $2 \sigma$ sensitivity and the dashed blue curve shows the projected $5 \sigma$ sensitivity for the JEF experimental reach. Dashed gray contours denote the upper bound on the mass scale $\Lambda$ for new electroweak fermions needed for anomaly cancellation.

left-right asymmetry in the Dalitz distribution [33, 64]. These measurements will provide the best direct constraints on new $\mathrm{C}$ violating, $\mathrm{P}$ conserving interactions.

\subsection{Probe the scalar dynamics in ChPT}

The SM allowed $\eta \rightarrow \pi^{0} \gamma \gamma$ is the only known meson decay which proceeds via a polarizability type of mechanism. It offers a unique opportunity to test high order ChPT [65]. Since $\eta$ and $\pi^{0}$ being neutral, tree-level contributions to $\eta \rightarrow \pi^{0} \gamma \gamma$ vanish both at $\mathscr{O}\left(p^{2}\right)$ and at $\mathscr{O}\left(p^{4}\right)$ in the chiral expansion. The first non-vanishing contributions are the meson loops at $\mathscr{O}\left(\hat{p}^{4}\right)$ with either $\pi^{+} \pi^{-}$ or $K^{+} K^{-}$intermediate states. However, rescattering $\pi^{+} \pi^{-} \rightarrow \pi^{0} \eta$ requires isospin breaking and is strongly suppressed, while kaon loops turn out to be small due to combinatorial factors and the large kaon mass in the denominator [66]. The major contributions to $\eta \rightarrow \pi^{0} \gamma \gamma$ are two $\mathscr{O}\left(p^{6}\right)$ counter- 
terms in the chiral Lagrangian [66]. This unique window for high order ChPT has inspired many theoretical and experimental studies in the past half century [68]. The proposed JEF experiment will measure the $\eta \rightarrow \pi^{0} \gamma \gamma$ branching ratio and the Dalitz distribution as shown in Fig. 9 and Fig. 10, providing a model-independent determination of two $\mathscr{O}\left(p^{6}\right)$ Low Energy Constants (LEC).

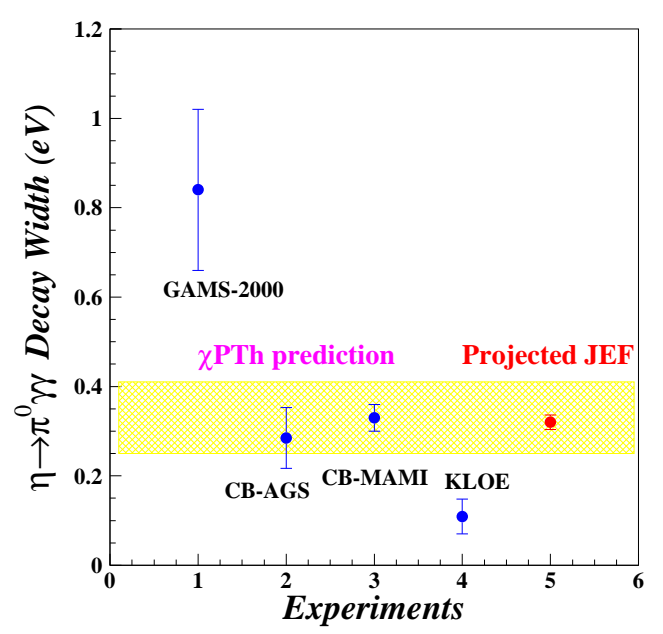

Figure 9: Experimental results on the decay width of $\eta \rightarrow \pi^{0} \gamma \gamma[69,70,71,72]$. The yellow band is $\Gamma=(0.33 \pm 0.08) \mathrm{eV}$ from the most recent unitary ChPT calculation by Oset et al. [73]. Projected JEF measurement with a total error of 5\% (in red) is arbitrarily plotted at the CB-MAMI value [71].

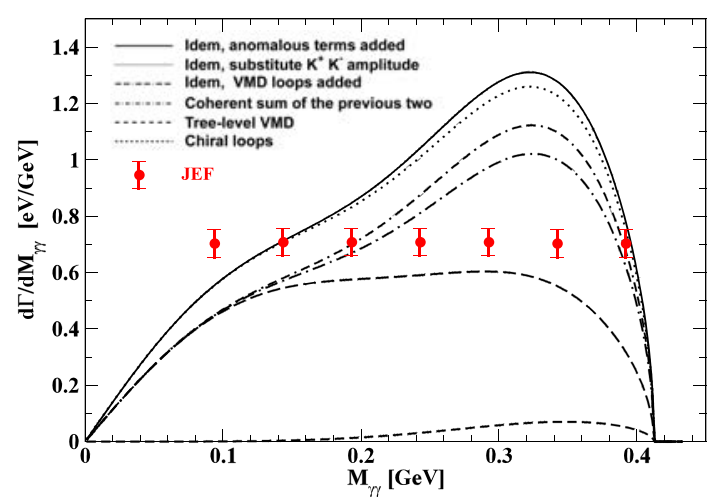

Figure 10: Predicted two-photon invariant mass distributions from $\eta \rightarrow \pi^{0} \gamma \gamma$ due to different mechanisms [73] and the projected JEF measurement with 100 days of beam time.

The physics impact of $\eta \rightarrow \pi^{0} \gamma \gamma$ goes far beyond these two LEC's [65]. There are 56-94 LEC's at $\mathscr{O}\left(p^{6}\right)$, depending on the numbers of flavors included in the Lagrangian. Some combinations of those LEC's are known from curvature of the scalar and vector form factors and from $\pi \pi$ scattering, but the only option to determine so many LEC's will be calculation rather than experiment. The most widely used theoretical approach to calculate LEC's uses the meson resonance approximation. Vector Meson Dominance (VMD) has been tested and is well understood [67]; however, the scalar meson contributions are poorly known since they contribute mostly where loop contributions are also important. The decay of $\eta \rightarrow \pi^{0} \gamma \gamma$ has a unique feature that the shape of the two-photon invariant mass spectrum, $d \Gamma / d M_{\gamma \gamma}$, is sensitive to the role of scalar resonances [74]. As clearly seen in Fig. 10, the predicted full distribution including the scalar contributions(solid line) has a significant enhancement at higher diphoton invariant masses, as compared to the flatter pure VMD prediction (long-dashed). Therefore a precision measurement of this distribution offers a clean window to understand the interplay of meson resonances at $\mathscr{O}\left(p^{6}\right)$ ChPT [26]. The projected JEF precision, shown in Fig. 10, would be sufficient to determine the scalar-VMD interference contribution and distinguish it from the VMD mechanism alone for the first time. It would provide a sensitive probe to test the ability of models such as meson resonance saturation to calculate many other unknown $\mathscr{O}\left(p^{6}\right)$ Low Energy Constants. 


\subsection{Precision determination of the quark mass ratio}

Reduction of the uncertainty on the quark mass ratio, $\mathscr{Q} \equiv\left(m_{s}^{2}-\hat{m}^{2}\right) /\left(m_{d}^{2}-m_{u}^{2}\right)$ with $\hat{m} \equiv$ $\left(m_{u}+m_{d}\right) / 2$, will be achieved by a high statistics measurement in the Dalitz distributions of $\eta \rightarrow 3 \pi$ while controlling systematic uncertainties due to relatively flat acceptance and detection efficiency over phase space.

As discussed in the previous section 3, the experimental Dalitz distributions for $\eta \rightarrow 3 \pi$ help to constrain the theoretical calculation of $\mathscr{M}(s, t, u)$ in order to extract the quark mass ratio. There have been several experimental results published in the recent years from the KLOE, Crystal Ball, and WASA collaborations $[75,76,77,78]$. The results for the slope parameter, $\alpha$, in $\eta \rightarrow 3 \pi^{0}$ are consistent among those measurements. Compared to the charged decay channel, the neutral decay channel provides weaker constraint on theory due to identical final state particles. On the other hand, the current experimental results from the charged channel still have significant discrepancies. The existing experiments were performed with unboosted $\eta$ 's. The energy of the decay particles from $\eta$ 's were relatively small hence the detection efficiency was more sensitive to the detector threshold. The JEF experiment will collect about 20 million of $\eta \rightarrow 3 \pi$ events for both neutral and charged channels. The highly boosted $\eta$ 's produced by a $12 \mathrm{GeV}$ photon beam will be less sensitive to the detection threshold and will offer a new result with significantly different systematics in addition to higher statistics. Such cross checks on the systematics are important for understanding the uncertainty on the quark mass ratio $\mathscr{Q}$, a fundamental QCD parameter.

\subsection{Status of the JEF project}

The JEF proposal [36] was submitted to Jlab Program Advisory Committee (PAC) in 2014. The non-rare $\eta \rightarrow 3 \pi$ decay can run in parallel to the approved GlueX and PrimEx-eta (E12-10011) experiments using the standard GlueX apparatus. PAC considered it as "the most compelling physics result and recommends to perform this measurement as a run group with GlueX and experiment E12-10-011" [79]. The GlueX apparatus has been successfully constructed and it was commissioned in fall 2014 and spring 2015. The physics runs will begin in spring 2016. The rare $\eta$ decays require an upgraded FCAL-II. PAC conditionally approved the rare decay part of physics with a recommendation that "the impact of a discovery in the proposed channels would be enormous, so as not to prevent these studies from running in the near future, we therefore ask that FCAL-II and the associated JEF physics program be fully incorporated to run in parallel with GlueX" [79]. The collaboration is working intensively to explore all possibilities to run the rare decay part JEF program in parallel to the GlueX experiment with an upgraded FCAL-II. An updated JEF proposal will be submitted to PAC in 2016.

\section{Summary}

The experimental programs on precision measurements of light pseudoscalar mesons $\pi, \eta$ and $\eta^{\prime}$ have been developed at Jlab. These programs will provide an unprecedented opportunity to understand the confinement QCD and search for new physics beyond the Standard Model. 


\section{References}

[1] J.S.Bell and R.Jaciw, Nuovo Cimento A 60, 47 (1969); S.L.Adler, Phys. Rev. 177, 2426 (1969).

[2] A.M. Bernstein and B.R. Holstein, Rev. Mod. Phys., 85, 49 (2013).

[3] K. Nakamura et al. (Particle Data Group), J. Phys. G 37, 075021 (2010).

[4] J. L. Goity, A.M. Bernstein, B.R. Holstein, Phys. Rev. D 66, 076014 (2002).

[5] B. Ananthanarayan and B. Moussallam, JHEP 0205, 052 (2002).

[6] K. Kampf and B. Moussallam, Phys. Rev. D 79, 076005 (2009).

[7] B. L. Ioffe and A. G. Oganesian, Phys. Lett. B 647, 389 (2007).

[8] R.M.Barnett et al., Review of Particle Physcis, Phys. Rev. D 54, 1 (1996).

[9] H.W. Atherton et al., Phys. Lett. B 158, 81 (1985).

[10] A. Browman et al., Phys. Rev. Lett. 33, 1400 (1974).

[11] G. Bellettini et al., Il Nuova Cimento, 66, 243 (1970).

[12] V.I. Kryshkin et al., Sov. Phys. JETP, 30, 1037 (1970).

[13] I. Larin et al. (the PrimEx collaboration), Phys. Rev. Lett., 106, 162303 (2011).

[14] H. Primakoff, Phys. Rev. 81, 899 (1951).

[15] L. Gan, EPJ Web Conf. 73, 07004 (2014).

[16] Jefferson Lab $12 \mathrm{GeV}$ upgrade white paper, "Science Driving the 12 GeV CEBAF Upgrade”, 2001.

[17] K.A. Olive et al. (Particle Data Group), Chin. Phys. C 38, 090001 (2014).

[18] S.E. Baru et al., Z. Phys. C 48, 581 (1990).

[19] N.A. Roe et al., Phys. Rev. D 41, 17 (1990).

[20] D.A. Williams et al., Phys. Rev. D 38, 1365 (1988).

[21] W. Bartel et al., Phys. Lett. B 160, 421 (1985).

[22] D. Babusci et al., J. High. Ener. Phys. 1301, 119 (2013).

[23] A. Gasparian and L. Gan et al., Jlab proposal "A Precision Measurement of the $\eta$ Radiative Decay Width via the Primakoff Effect”, https://www.jlab.org/exp_prog/proposals/10/PR12-10-011.pdf.

[24] L. Gan, PoS CD12, 117 (2013).

[25] H. Leutwyler, Phys. Lett. B 374, 181 (1996).

[26] J. Bijnens and J. Gasser, Proceeding for the workshop on eta physics, Uppasala, October 25-27, 2001.

[27] A. Browman et al., Phys. Rev. Lett. 32, 1067 (1974).

[28] V. Pauk and M. Vanderhaeghen, Eur. Phys. J. C 74, 3008 (2014).

[29] M. Knecht and A. Nyffeler, Phys. Rev. D 65, 073034 (2002).

[30] H.J. Behrend, et, al., Z. Phys. C 49, 401 (1991).

[31] J. S. Bell and D. G. Sutherland, Nucl. Phys. B 4, 315 (1968).

[32] D. G. Sutherland, Phys. Lett. 23, 384 (1966). 
[33] Workshop on "Hadronic Probes of Fundamental Symmetries", the Amherst Center for Fundamental Interactions (ACFI), University of Massachusetts, Amherst, MA, March 6-8, 2014. https://www.physics.umass.edu/acfi/seminars-and-workshops/hadronic-probes-of-fundamentalsymmetries.

[34] E. Passemar, talk at ACFI workshop "Hadronic Probes of Fundamental Symmetries”.

[35] L. Gan and A. Gasparian, PoS CD09, 048 (2009).

[36] L. Gan et al., Jlab proposal "Eta Decays with Emphasis on Rare Neutral Modes: The JLab Eta Factory (JEF) Experiment”, https://www.jlab.org/exp_prog/proposals/14/PR12-14-004.pdf.

[37] E.L. Bratkovskaya et al., Phys. Lett. B 359, 217 (1995).

[38] P. Langacker, Rev. Mod. Phys. 81, 1199 (2009).

[39] G.R. Farrar and G. Zaharijas, Phys. Rev. Lett. 96, 041302 (2006).

[40] M. Duerr and P.F. Perez, Phys. Lett. B 732, 101 (2014).

[41] H. Davoudiasl et al., Phys. Rev. Lett. 105, 211304 (2010)

[42] K. Agashe and G. Servant, Phys. Rev. Lett. 93, 231805 (2004)

[43] M.L. Graesser, I.M. Shoemaker, L. Vecchi, arXiv:1107.2666.

[44] B. Batell et al., Phys. Rev. D 90, 115014 (2014).

[45] B. Holdom, Phys. Lett. B 166, 196 (1986).

[46] R. Essig et al., arXiv:1311.0029.

[47] A. E. Nelson and N. Tetradis, Phys. Lett. B 221, 80 (1989).

[48] S. Tulin, Phys. Rev. D 89, 114008 (2014).

[49] T.D. Lee and C.N. Yang, Phys. Rev. 98, 1501 (1955).

[50] R. Foot, G. C. Joshi, and H. Lew, Phys. Rev. D 40, 2487 (1989).

[51] S. Rajpoot, Phys. Rev. D 40, 2421 (1989); X.-G. He and S. Rajpoot, Phys. Rev. D 41, 1636 (1990); C. D. Carone and H. Murayama, Phys. Rev. Lett. 74, 3122 (1995); D. C. Bailey and S. Davidson, Phys. Lett. B 348, 185 (1995); C. D. Carone and H. Murayama, Phys. Rev. D 52, 484 (1995); A. Aranda and C. D. Carone, Phys. Lett. B 443, 352 (1998); P. Fileviez Perez and M. B. Wise, Phys. Rev. D 82, 011901 (2010).

[52] B. Dobrescu and C. Frugiuele, Phys. Rev. Lett. 113, 061801 (2014).

[53] T. Cohen et al., Phys. Rev. Lett. 115, 171804 (2015).

[54] H. Davoudiasl and R.N. Mohapatra, New J. Phys. 14, 095011 (2012); K.M. Zurek, Phys. Rept. 537, 91 (2014); K. Petraki and R.R. Volkas, Int. J. Mod. Phys. A 28, 1330028 (2013).

[55] M. Williams, C. Burgess, A. Maharana, and F. Quevedo, JHEP 1108, 106 (2011).

[56] J. Beringer et al., (Particle Data Group), Phys. Rev. D 86, 010001 (2012).

[57] A. Aranda and C. D. Carone, Phys. Lett. B 443, 352 (1998).

[58] R. Barbieri and T. E. O. Ericson, Phys. Lett. B 57, 270 (1975).

[59] D. Babusci et al., Phys. Lett. B 720, 111 (2013).

[60] P. Adlarson et al., Phys. Lett. B 726, 187 (2013). 
[61] B.M.K. Nefkens et al., Phys. Rev. C 72, 035212 (2005).

[62] M.J. Ramsey-Musolf, talk at ACFI workshop on "Hadronic Probes of Fundamental Symmetries".

[63] A. Kurylov, G. Mclaughlin and M. Ramsey-Musolf, Phys. Rev., D 63, 076007 (2001).

[64] S. Gardner, talk at ACFI workshop on“Hadronic Probes of Fundamental Symmetries”.

[65] J. Bijnens, talk at ACFI workshop on "Hadronic Probes of Fundamental Symmetries".

[66] L. Ametller, J. Bijnens, and F. Cornet, Phys. Lett., B 276, 185 (1992).

[67] B. Holstein, discussion at ACFI workshop on "Hadronic Probes of Fundamental Symmetries".

[68] M. N. Achasov, et, al., Nucl. Phys., B 600, 3 (2001).

[69] D. Alde et al., Yad. Fiz 40, 1447 (1984); D. Alde et al., Z. Phys. C 25, 225 (1984); L. G. Landsberg, Phys. Rep. 128, 301 (1985).

[70] S. Prakhov et al., Phys. Rev. C 78, 015206 (2008).

[71] B.M.K. Nefkens et al., Phys. Rev. C 90, 025206 (2014).

[72] B.Di Micco et al., Acta Phys. Slovaca 56, 403 (2006).

[73] E. Oset, J. R. Peláez, L. Roca, Phys. Rev. D 77, 073001 (2008); E. Oset, J. R. Peláez and L. Roca, Phys. Rev. D 67, 073013 (2003).

[74] L. Ametller, Phys. Scripta, T 99, 45 (2002).

[75] S. Prakhov et al., Phys. Rev. C 79, 035204 (2009).

[76] M. Unverzagt et al., Eur. Phys. J. A 39, 169 (2009).

[77] C. Adolph et al., Phys. Lett. B 677, 24, (2009).

[78] F. Ambrosino et al., Phys. Lett. B 694, 16 (2010).

[79] Jefferson Lab PAC42 report, 2014. https://www.jlab.org/exp_prog/PACpage/PAC42/PAC42_FINAL_Report.pdf 